\title{
Aplicación del Método Espectral a la Ecuación de Aguas Poco Profundas
}

\author{
Juan Luna Valdez ${ }^{1}$, Edith Carhuapoma Lopez $z^{2}$
}

Resumen: En este trabajo se estudia el sistema de ecuaciones de aguas poco profundas en la forma Lagrangiana y se obtiene su solución analítica. Aplicaremos el método espectral en el análisis numérico de este sistema y se muestra que la propagación de las ondas de las aguas poco profundas no dependen del medio en que se propaga.

Palabras clave: Ecuación de aguas poco profundas; Ley de conservación; Método espectral; Operador de la derivada material.

\section{Application of the Spectral Method to the Equation of Shallow Waters.}

Abstract: In this work we study the system of shallow water equations in the Lagrangian form and obtain its analytical solution. We apply the spectral method in the numerical analysis of this system and it is shown that the propagation of shallow water waves do not depend on the medium in which it propagates.

Keywords: Shallow water equation; Law of conservation; Spectral method; Operator of the material derivative

Recibido: 14/04/2017. Aceptado: 07/07/2017. Publicado online: 01/09/2017

(C)Los autores. Este artículo es publicado por la Revista PESQUIMAT de la Facultad de Ciencias Matemáticas, Universidad Nacional Mayor de San Marcos. Este es un artículo de acceso abierto, distribuido bajo los términos de la licencia Creative Commons Atribucion-No Comercia-Compartir Igual 4.0 Internacional. (http://creativecommons.org/licenses/by-nc-sa/4.0/) que permite el uso no comercial, distribución y reproducción en cualquier medio, siempre que la obra original sea debidamente citada. Para información, por favor póngase en contacto con revistapesquimat.matematica@unmsm.edu.pe

\footnotetext{
${ }^{1}$ UNMSM, Facultad de Ciencias Matemáticas, e-mail: jlunav@unmsm.edu.pe

${ }^{2}$ UNMSM, Facultad de Ciencias Matemáticas, e-mail:1410391@unmsm.edu.pe
} 


\section{Introducción}

Los fenómenos ligados al movimiento de los fluidos son estudiados por la mecánica de los fluidos, cuyas ecuaciones gobernantes son modelados matematicamente por medio de las leyes de conservación, las cuales consisten de un sistema de ecuaciones diferenciales parciales. La dificultad para encontrar las soluciones analíticas para estas ecuaciones, se debe al dominio irregular, la no linealidad de las ecuaciones, entre otros factores. Por eso, la necesidad del tratamiento numérico de estas ecuaciones, para las cuales usaremos el método espectral, ver [1, pág. 15] .

La dinámica de un fluido incompresible es descrita por la ley de conservación de masa y por la ley de conservación de momento, ver [2, pág. 1-15].

Las ecuaciones de aguas poco profundas forman un sistema de ecuaciones diferenciales parciales deducidos a partir de las leyes de conservación de masa y de momento de un fluido. Estas constituyen las conocidas ecuaciones de Euler de la dinámica de fluidos, las ecuaciones son un caso particular de las clásicas ecuaciones de Navier-Stokes.

En este trabajo deduciremos la forma Lagrangeana de las ecuaciones de aguas poco profundas, las cuales serán linealizados y estudiados en un dominio periódico. Luego aplicamos el método espectral en el análisis numérico de las ecuaciones de aguas poco profundas y mostramos que la velocidad de propagación de las ondas no dependen de la red que es usado para modelar el medio en que ellas se propagan.

\section{Ecuaciones de aguas poco profundas}

Consideremos para cualquier movimiento del fluido que la única fuerza ejercida sobre su superficie es la presión. Siendo el geopotencial (energía que no realiza trabajo) dado por $\phi=g h$, con $g$ el valor de la gravedad y $h=h(x, y)$ la altura del fluido, la fuerza ejercida sobre el movimiento del fluido es dado por medio del gradiente del mismo. En el modelo de aguas poco profundas, consideremos un fluido incompresible homogéneo con una frontera horizontal inferior rígida y superior una superficie libre. El campo de volocidades considerado será de velocidad horizontal lo cual es invariante con relación a la altura, esto es, $v_{H}=(u(x, y, z), v(x, y, z), t)$. En el sistema de aguas poco profundas, usamos las ecuaciones de conservación de momento y de masa (continuidad). La ecuación de continuidad para un fluido incompresible cualquiera, cuyo campo de velocidades es $\vec{U}(x, y, z)=(u(x, y, z), v(x, y, z), t)$ es dado por,

$$
\operatorname{div} \vec{U}=\frac{\partial u}{\partial x}+\frac{\partial v}{\partial y}+\frac{\partial w}{\partial z}=0
$$

integrando verticalmente esta ecuación, de $z=0$ hasta $z=h$, y con la condición de frontera de $w$ dado por $w(0)=0$ y $w(h)=w$, se tiene,

$$
\int_{0}^{h} \frac{\partial w}{\partial z} d z=-\int_{0}^{h}\left(\frac{\partial u}{\partial x}+\frac{\partial v}{\partial y}\right) d z
$$

esto es

$$
w=-h\left(\frac{\partial u}{\partial x}+\frac{\partial v}{\partial y}\right) \Longrightarrow w=-h\left(\operatorname{div} v_{H}\right) .
$$

Por otro lado, la velocidad de la partícula en la dirección de $z$ está dado por

$$
w=\frac{D h}{D t}=\frac{\partial h}{\partial t}+\left(v_{H} \cdot \nabla\right) h,
$$

donde

$$
\frac{\partial h}{\partial t}=-\left(v_{H} \cdot \nabla\right) h-h\left(\operatorname{div} v_{H}\right)
$$


que multiplicando por $g$ nos da la ecuación de continuidad de un fluido incompresible,

$$
\frac{\partial \phi}{\partial t}=-\left(v_{H} \cdot \nabla\right) \phi-\phi\left(\operatorname{div} v_{H}\right)
$$

de donde se tiene que

$$
\frac{D \phi}{D t}+\phi\left(\operatorname{div} v_{H}\right)=0
$$

Observación 2.1 Ley de Conservación de Masa (forma diferencial)

$$
\frac{D \rho}{D t}+\rho(\operatorname{div} U)=0
$$

donde $\rho$ es la densidad del fluido en un tiempo t cualquiera.

Además, se tiene la ecuación de conservación de movimiento

$$
\rho \frac{D u}{D t}=-\nabla p
$$

donde $p$ es la presión resultante de las fuerzas actuantes en la región dada.

Mas detalles, ver [2.pp. $1-11]$.

De la observación 2.1 el balance del momento horizontal dado en la ecuación de conservación de momento con $f=-\rho \nabla \phi$ es

$$
\frac{D v_{H}}{D t}+\nabla \phi=0
$$

Asi mismo, obtenemos el siguiente sistema de ecuaciones de aguas poco profundas

$$
\left\{\begin{array}{c}
\frac{D v_{H}}{D t}+\nabla \phi=0 \\
\frac{D \phi}{D t}+\phi\left(\operatorname{div} v_{H}\right)=0
\end{array}\right.
$$

de este modo, la primera ecuación de (4) puede ser escrita en la forma

$$
\frac{D v_{H}}{D t}+\nabla \phi=\left(u \frac{\partial u}{\partial x}+v \frac{\partial u}{\partial y}+\frac{\partial u}{\partial t}, u \frac{\partial v}{\partial x}+v \frac{\partial v}{\partial y}+\frac{\partial v}{\partial t}\right)+\left(\frac{\partial \phi}{\partial x}, \frac{\partial \phi}{\partial y}\right)=(0,0),
$$

esto proporciona

$$
\frac{D v_{H}}{D t}+\nabla \phi=\left(\frac{D u}{D t}, \frac{D v}{D t}\right)+\left(\frac{\partial \phi}{\partial x}, \frac{\partial \phi}{\partial y}\right)=(0,0),
$$

y la segunda ecuación del sistema (4) es

$$
\frac{D \phi}{D t}+\phi\left(\frac{\partial u}{\partial x}+\frac{\partial v}{\partial y}\right)=0
$$

Consideremos un vector onda $c=\left(c_{x}, c_{y}\right)$ que es un vector que apunta en la dirección de la propagación de la onda en cuestión y cuya magnitud es el número de onda. Ahora introduciendo artificialmente el vector de onda $c=\left(c_{x}, c_{y}\right)$, para analizar el efecto de la red heterogéneo en la propagación de ondas de aguas poca profundas en el sistema (4), reordenado el sistema, se tiene

$$
\left\{\begin{aligned}
\frac{D u}{D t}+c_{x}^{2} \frac{\partial \phi}{\partial x} & =0 \\
\frac{D v}{D t}+c_{y}^{2} \frac{\partial \phi}{\partial y} & =0 \\
\frac{D \phi}{D t}+\phi\left(\frac{\partial u}{\partial x}+\frac{\partial v}{\partial y}\right) & =0
\end{aligned}\right.
$$


Desdoblando la primera ecuación del sistema (5), se tiene

$$
\frac{\partial u}{\partial t}+u \frac{\partial u}{\partial x}+v \frac{\partial u}{\partial y}+c_{x}^{2} \frac{\partial \phi}{\partial x}=0 .
$$

Antes de continuar con nuestro estudio vamos dar algunas definiciones importantes del estado estacionario.

Linealizando el sistema (5) en torno de un estado estacionario de $u=U, v=V, \phi=\Phi$, estas constantes, se puede escribir como

$$
u=U+u^{\prime}, v=V+v^{\prime}, \phi=\Phi+\phi^{\prime},
$$

donde $u^{\prime}, v^{\prime}, \phi^{\prime}$ son desviaciones de $O(\epsilon)$ con $\epsilon<<1$, del estado estacionario.

Luego, por (6)

$$
\frac{\partial\left(U+u^{\prime}\right)}{\partial t}+\left(U+u^{\prime}\right) \frac{\partial\left(U+u^{\prime}\right)}{\partial x}+\left(V+v^{\prime}\right) \frac{\partial\left(U+u^{\prime}\right)}{\partial y}+c_{x}^{2} \frac{\partial\left(\Phi+\phi^{\prime}\right)}{\partial x}=0,
$$

como $(U, V, \Phi)$ es constante entonces sus derivadas son nulas. Luego la ecuación anterior queda

$$
\frac{\partial u^{\prime}}{\partial t}+U \frac{\partial u^{\prime}}{\partial x}+u^{\prime} \frac{\partial u^{\prime}}{\partial x}+V \frac{\partial u^{\prime}}{\partial y}+v^{\prime} \frac{\partial u^{\prime}}{\partial x}+c_{x}^{2} \frac{\partial \phi^{\prime}}{\partial x}=0
$$

tenemos tambien que, $\frac{\partial u^{\prime}}{\partial x}, u^{\prime}, \frac{\partial u^{\prime}}{\partial y}, v^{\prime}$ son $O(\epsilon)$, entonces el producto entre ellos es de orden $O\left(\epsilon^{2}\right)$, es decir, despreciable. Además, se tiene la siguiente ecuación con terminos $O(\epsilon)$,

$$
\frac{\partial u^{\prime}}{\partial t}+U \frac{\partial u^{\prime}}{\partial x}+V \frac{\partial u^{\prime}}{\partial y}+c_{x}^{2} \frac{\partial \phi^{\prime}}{\partial x}=0
$$

De forma análoga, se hace para la segunda y tercera ecuación del sistema (5), y volviendo a denotar los términos $u, v, \phi$, se tiene el siguiente sistema

$$
\left\{\begin{aligned}
\frac{\partial u}{\partial t}+U \frac{\partial u}{\partial x}+V \frac{\partial u}{\partial y}+c_{x}^{2} \frac{\partial \phi}{\partial x} & =0 \\
\frac{\partial v}{\partial t}+U \frac{\partial v}{\partial x}+V \frac{\partial v}{\partial y}+c_{y}^{2} \frac{\partial \phi}{\partial y} & =0 \\
\frac{\partial \phi}{\partial t}+U \frac{\partial \phi}{\partial x}+V \frac{\partial \phi}{\partial y}+\Phi\left(\frac{\partial u}{\partial x}+\frac{\partial u}{\partial y}\right) & =0
\end{aligned}\right.
$$

donde $0 \leq x \leq L_{x}$ y $0 \leq y \leq L_{y}$. Las condiciones de contorno que serán considerados son periódicas tanto en la dirección $x$ como en la dirección $y$. Consideremos la solución de (7) de la forma

$$
\left(\begin{array}{l}
u \\
y \\
\phi
\end{array}\right)(x, y, t)=\sum_{k=-\infty}^{\infty} \sum_{l=-\infty}^{\infty} \alpha(k, l) e^{i\left(w_{x} k x+w_{y} l y-w t\right)}\left(\begin{array}{c}
u_{k, l}^{0} \\
v_{k, l}^{0} \\
\phi_{k, l}^{0},
\end{array}\right)
$$

donde denotamos la frecuencia temporal por $w$, las frecuencias espaciales por

$$
w_{x}=\frac{2 \pi}{L_{x}}, \quad w_{y}=\frac{2 \pi}{L_{y}}
$$

y siendo $u_{k, l}^{0}, v_{k, l}^{0}$ y $\phi_{k, l}^{0}$ las condiciones iniciales que son dadas. 
Reemplazando esta solución en (7) y dejando los subindices $k$ y $l$ en $u_{k, l}^{0}, v_{k, l}^{0}, \phi_{k, l}^{0}$ obtenemos (haciendo las debidas simplificacciones),

$$
\left(\begin{array}{ccc}
-w+U w_{x} k+V w_{y} l & 0 & c_{x}^{2} w_{x} k \\
0 & -w+U w_{x} k+V w_{y} l & c_{y}^{2} w_{y} l \\
\Phi w_{x} k & \Phi w_{y} l & -w+U w_{x} k+V w_{y} l
\end{array}\right)\left(\begin{array}{l}
u^{0} \\
v^{0} \\
\phi^{0}
\end{array}\right)=\left(\begin{array}{l}
0 \\
0 \\
0
\end{array}\right) .
$$

Introducimos la notación

$$
G_{k, l}=\sqrt{\Phi\left[c_{x}^{2}\left(w_{x} k\right)^{2}+c_{y}^{2}\left(w_{y} l\right)^{2}\right]} .
$$

Se quiere soluciones no triviales para el sistema de aguas poco profundas. De este modo, anulando el determinante de la matriz (8) resulta la siguiente ecuación cúbica

$$
\left(-w+U w_{x} k+V w_{y} l\right)\left[\left(-w+U w_{x} k+V w_{y} l\right)^{2}-G_{k, l}^{2}\right]=0 .
$$

Una de las raices de la ecuación (10) es

$$
w_{0}=U w_{x} k+V w_{y} l .
$$

Para $w \neq U w_{x} k+V w_{y} l$ se obtiene al extraer el valor de $w$ en la ecuación (10)

$$
w_{+}=U w_{x} k+V w_{y} l+G_{k, l}
$$

$\mathrm{y}$

$$
w_{-}=U w_{x} k+V w_{y} l-G_{k, l} .
$$

El autovector correspondiente al autovalor dado en (11) es solución de

$$
\left(\begin{array}{ccc}
0 & 0 & c_{x}^{2} w_{x} k \\
0 & 0 & c_{y}^{2} w_{y} l \\
\Phi w_{x} k & \Phi w_{y} l & 0
\end{array}\right)\left(\begin{array}{l}
u^{0} \\
v^{0} \\
\phi^{0}
\end{array}\right)=\left(\begin{array}{l}
0 \\
0 \\
0
\end{array}\right)
$$

Si $k \neq 0$ o $l \neq 0$ entonces, sin pérdida de generalidad, supongamos que $k \neq 0$, pues el caso que $l \neq 0$ es análogo, luego se tiene

$$
\left\{\begin{array}{ccc}
\phi^{0} & = & 0 \\
w_{x} k u^{0}+w_{y} l v^{0} & = & 0
\end{array} \Longrightarrow u^{0}=-\frac{w_{y} l}{w_{x} k} v^{0}, \quad k \neq 0 .\right.
$$

Además, se tiene el autovector

$$
R_{0}(k, l)=\left(-w_{y} l, w_{x} k, 0\right)^{T} .
$$

El autovector para el autovalor $w_{+}$dado en (12) es solución de

$$
\left(\begin{array}{ccc}
-G_{k, l} & 0 & c_{x}^{2} w_{x} k \\
0 & -G_{k, l} & c_{y}^{2} w_{y} l \\
\Phi w_{x} k & \Phi w_{y} l & -G_{k, l}
\end{array}\right)\left(\begin{array}{c}
u^{0} \\
v^{0} \\
\phi^{0}
\end{array}\right)=\left(\begin{array}{l}
0 \\
0 \\
0
\end{array}\right)
$$

luego,

$$
\left\{\begin{array}{cc}
G_{k, l} u^{0}-c_{x}^{2} w_{x} k \phi^{0} & =0 \\
G_{k, l} v^{0}-c_{y}^{2} w_{y} l \phi^{0} & =0 \\
-w_{x} k \Phi u^{0}-w_{y} l \Phi v^{0}+G_{k, l} \Phi^{0} & =0
\end{array}\right.
$$


y las dos primeras ecuaciones anteriores nos da

$$
\left\{\begin{array}{l}
u^{0}=\frac{c_{x}^{2} w_{x} k}{G_{k, l}} \phi^{0} \\
v^{0}=\frac{c_{y}^{2} w_{y} k}{G_{k, l}} \phi^{0}
\end{array}\right.
$$

lo que nos da el autovector

$$
R_{+}(k, l)=\left(c_{x}^{2} w_{x} k, c_{y}^{2} w_{y} l, G_{k, l}\right)^{T} .
$$

De forma análoga, para el autovalor $w_{-}$dado en (13) se tiene el autovector

$$
R_{-}(k, l)=\left(c_{x}^{2} w_{x} k, c_{y}^{2} w_{y} l,-G_{k, l}\right)^{T} .
$$

Ahora, si $k l=0$, por ejemplo, supongamos que $k=0$ y $l \neq 0$, pues el caso $k \neq 0$ y $l=0$ es análogo, entonces por (15) se tiene que $R_{0}=(1,0,0)^{T}$ y por $(17)$, se tiene que $R_{+}(0, l)=$ $\left(0, c_{y}^{2} w_{y} l, G_{0, l}\right)^{T} \quad$ y análogamente $R_{-}(0, l)=\left(0, c_{y}^{2} w_{y} l,-G_{0, l}\right)^{T}$.

Si $k=l=0$ en (15), (17) y (18), entonces los autovalores $w_{0}, w_{+}, w_{-}$y autovectores $R_{0}, R_{+}, R_{-}$ son nulos. Además no hay interés físico pues ellos no dependen de los números de ondas.

Por tanto, se demuestra el siguiente resultado

Teorema 2.2 La solución general no estacionario del sistema (7) puede ser escrita en la forma

$$
\begin{gathered}
S(x, y, t)=\sum_{k=-\infty}^{\infty} \sum_{\substack{l=-\infty \\
(k, l) \neq(0,0)}}^{\infty}\left[\alpha_{+}(k, l) e^{i\left(w_{x} k x+w_{y} l y-w_{+} t\right)} R_{+}+\alpha_{-}(k, l) e^{i\left(w_{x} k x+w_{y} l y-w_{-} t\right)} R_{-}\right. \\
\left.+\alpha_{0}(k, l) e^{i\left(w_{x} k x+w_{y} l y-w_{0} t\right)} R_{0}\right]
\end{gathered}
$$

donde $w_{+}, w_{-}, w_{0}$ son autovalores y $R_{+}(k, l), R_{-}(k, l), R_{+}(k, l)$ son los respectivos autovectores del sistema matricial (8) y $S=(u, v, \phi)^{T}$.

La solución del sistema (7) correspondientes a los indices $k=0, l=0$ es nula, por lo tanto es estacionaria, luego no sufre interferencia del medio y no tiene interés físico. Por tanto no será analizado, por que ahora estamos interesados en la solución de la perturbación del sistema (4) en torno del estado estacionario $(U, V, \Phi)$.

Los autovectores $R_{+}$y $R_{-}$correspondientes a las ondas de gravedad (ondas rápidas) es $R_{0}$ que corresponde a la onda de Rossby (onda lenta). Ver [3].

El problema ahora, es encontrar los coeficientes $\alpha_{+}, \alpha_{-}, \alpha_{0}$. Estos pueden ser determinados a partir de alguna condición inicial dada. Para la solución general dada en el teorema (2.2), se tiene.

$$
\left(\begin{array}{l}
u \\
v \\
\phi
\end{array}\right)(x, y, 0)=\sum_{k=-\infty}^{\infty} \sum_{\substack{l=-\infty \\
k, l) \neq(0,0)}}^{\infty} e^{i\left(w_{x} k x+w_{y} l y\right)}\left[\alpha_{+}(k, l) R_{+}+\alpha_{-}(k, l) R_{-}+\alpha_{0}(k, l) R_{0}\right] .
$$

Por la serie de Fourier se puede escribir las condiciones iniciales como,

$$
\left(\begin{array}{l}
u \\
v \\
\phi
\end{array}\right)(x, y, 0)=\sum_{k=-\infty}^{\infty} \sum_{\substack{l=-\infty \\
(k, l) \neq(0,0)}}^{\infty}\left(\begin{array}{c}
\widehat{u} \\
\widehat{v} \\
\widehat{\phi}
\end{array}\right)(x, y, 0) e^{i\left(w_{x} k x+w_{y} l y\right)} .
$$

De esta forma, se tiene la siguiente observación, 
Observación 2.3 La solución del sistema (7) es completamente determinada, dada la condición inicial $(u(x, y, 0), v(x, y, 0), \phi(x, y, 0))^{T}$.

En efecto, para $t=0, \mathrm{y} \quad(k, l) \neq(0,0)$ en la solución general dada en el teorema $(7)$, se tiene el siguiente sistema

$$
\left\{\begin{array}{cc}
{\left[\alpha_{+}(k, l)+\alpha_{-}(k, l)\right] c_{x}^{2} w_{x} k-\alpha_{0}(k, l) w_{y} l} & =\widehat{u}_{0} \\
{\left[\alpha_{+}(k, l)+\alpha_{-}(k, l)\right] c_{y}^{2} w_{y} l+\alpha_{0}(k, l) w_{x} k} & =\widehat{v}_{0} \\
{\left[\alpha_{+}(k, l)-\alpha_{-}(k, l)\right] G_{k, l}} & =\widehat{\phi}_{0}
\end{array}\right.
$$

donde los segundos miembros son los coeficientes de Fourier de los datos iniciales. Luego la solución de este sistema proporciona los coeficientes de Fourier $\alpha_{+}, \alpha_{-}, \alpha_{0}$.

\subsection{Análisis Espectral de las Ecuaciones de Aguas poco Profundas}

Aquí, analizaremos el efecto del uso de la red heterogéneas en la evolución de ondas de agua poco profundas planares utilizando el método espectral, que consiste en expandir las variables del sistema en series de Fourier. Nuestro objetivo es mostrar que la propagación de las ondas no sufre interferencia, por uso de redes heterogéneas, diferentemente del método de diferencias finitas, ver [4].

Consideremos el sistema (7) en el caso $U=V=0$, por que el término de advección de onda que se origina no es tratado espectralmente, pero si la parte lagrangeana. Cuando las variables $U, V$ son diferentes de cero, el método es conocido como Semi-Lagrangeano, y no será estudiado en este trabajo. Para una aplicación de de este método, ver [5]. Entonces discretizamos el sistema (7) usando diferencias finita progresiva en la derivada temporal

$$
\frac{\partial()}{\partial t}=\frac{()^{+}-()^{-}}{\Delta t}
$$

donde $\Delta t$ es el paso de tiempo, ()$^{+}$denota la evaluación en un punto de la cuadricula en tiempo $t+\Delta t$ y ()$^{-}$denota la evaluación en un punto de la cuadricula en tiempo $t$.

En las derivadas espaciales $u_{x}, u_{y}, \phi_{x}, \phi_{y}$, hacemos un tratamiento implícito de los términos lineales que producen las ondas de gravedad (ondas rápidas), usando el promedio temporal, que resultan en la siguiente discretización del sistema (7)

$$
\left\{\begin{array}{cc}
\frac{u^{+}-u^{-}}{\Delta t}+c_{x}^{2} \frac{\phi_{x}^{+}+\phi_{x}^{-}}{2} & =0 \\
\frac{v^{+}-v^{-}}{\Delta t}+c_{y}^{2} \frac{\phi_{y}^{+}+\phi_{y}^{-}}{2} & =0 \\
\frac{\phi^{+}-\phi^{-}}{\Delta t}+\Phi\left(\frac{u_{x}^{+}+u_{x}^{-}}{2}+\frac{v_{y}^{+}+v_{y}^{-}}{2}\right) & =0
\end{array}\right.
$$

Consideremos la solución numérica del esquema (20) de la forma

$$
\left(\begin{array}{c}
u^{-} \\
v^{-} \\
\phi^{-}
\end{array}\right)(x, y, t)=\sum_{k=-\infty}^{\infty} \sum_{l=-\infty}^{\infty} \alpha(k, l) e^{i\left(w_{x} k x+w_{y} l y-w t\right)}\left(\begin{array}{c}
u_{k, l}^{0} \\
v_{k, l}^{0} \\
\phi_{k, l}^{0}
\end{array}\right)
$$

y

$$
\left(\begin{array}{c}
u^{+} \\
v^{+} \\
\phi^{+}
\end{array}\right)(x, y, t)=\sum_{k=-\infty}^{\infty} \sum_{l=-\infty}^{\infty} \alpha(k, l) e^{i\left(w_{x} k x+w_{y} l y-w(t+\Delta t)\right)}\left(\begin{array}{c}
u_{k, l}^{0} \\
v_{k, l}^{0} \\
\phi_{k, l}^{0} .
\end{array}\right)
$$


Sustituyendo (21) y (22) en (20), cancelando el término $e^{i\left(w_{x} k x+w_{y} l y-w t\right)}$ y dejando los subindices $k, l$ en $u_{k, l}^{0}, v_{k, l}^{0}, \phi_{k, l}^{0}$ se tiene

$$
\left\{\begin{array}{cc}
\frac{e^{-i w \Delta t}-1}{\Delta t} u^{0}+\frac{e^{-i w \Delta t}+1}{2} i c_{x}^{2} w_{x} k \phi^{0} & =0, \\
\frac{e^{-i w \Delta t}-1}{\Delta t} v^{0}+\frac{e^{-i w \Delta t}+1}{2} i c_{y}^{2} w_{y} l \phi^{0} & =0 \\
\frac{e^{-i w \Delta t}-1}{\Delta t} \phi^{0}+\Phi \frac{e^{-i w \Delta t}+1}{2}\left(i w_{x} k u^{0}+i w_{y} l v^{0}\right) & =0 .
\end{array}\right.
$$

Multiplicando cada ecuación por $e^{\frac{i}{2} w \Delta t}$ se tiene

$$
\left\{\begin{array}{cl}
\frac{e^{-\frac{i}{2} w \Delta t}-e^{\frac{i}{2} w \Delta t}}{\Delta t} u^{0}+\frac{e^{-\frac{i}{2} w \Delta t}+e^{\frac{i}{2} w \Delta t}}{2} i c_{x}^{2} w_{x} k \phi^{0} & =0, \\
\frac{e^{-\frac{i}{2} w \Delta t}-e^{\frac{i}{2} w \Delta t}}{\Delta t} v^{0}+\frac{e^{-\frac{i}{2} w \Delta t}+e^{\frac{i}{2} w \Delta t}}{2} i c_{y}^{2} w_{y} l \phi^{0} & =0 \\
\frac{e^{-\frac{i}{2} w \Delta t}-e^{\frac{i}{2} w \Delta t}}{\Delta t} \phi^{0}+\Phi \frac{e^{-\frac{i}{2} w \Delta t}+e^{\frac{i}{2} w \Delta t}}{2}\left(i w_{x} k u^{0}+i w_{y} l v^{0}\right) & =0 .
\end{array}\right.
$$

Además, utilizando las definiciones del seno y coseno complejos, se tiene la siguiente forma para el sistema anterior,

$$
\left\{\begin{array}{cc}
\frac{2 i S}{\Delta t} u^{0}+C i c_{x}^{2} w_{x} k \phi^{0} & =0 \\
\frac{2 i S}{\Delta t} v^{0}+C i c_{y}^{2} w_{y} l \phi^{0} & =0 \\
\frac{2 i S}{\Delta t} \phi^{0}+\Phi C\left(i w_{x} k u^{0}+i w_{y} l v^{0}\right) & =0
\end{array}\right.
$$

donde

$$
S=\sin \left(-w \frac{\Delta t}{2}\right)
$$

y

$$
C=\cos \left(-w \frac{\Delta t}{2}\right)
$$

o la forma matricial, denotando $T=S / C$, se tiene

$$
\left(\begin{array}{ccc}
\frac{2 T}{\Delta t} & 0 & c_{x}^{2} w_{x} k \\
0 & \frac{2 T}{\Delta t} & c_{y}^{2} w_{y} l \\
\Phi w_{x} k & \Phi w_{y} l & \frac{2 T}{\Delta t}
\end{array}\right)\left(\begin{array}{c}
u^{0} \\
v^{0} \\
\phi^{0}
\end{array}\right)=\left(\begin{array}{l}
0 \\
0 \\
0
\end{array}\right) .
$$

Nuestro objetivo es encontrar soluciones no triviales para el sistema de ecuaciones de aguas poco profundas discretizadas. De esta forma, al igualar a cero el determinante del sistema (24), se tiene

$$
\left(\frac{2 T}{\Delta t}\right)^{3}-\left(\frac{2 T}{\Delta t}\right)\left[\Phi\left(c_{x}^{2}\left(w_{x} k\right)^{2}+c_{y}^{2}\left(w_{y} l\right)^{2}\right)\right]=0 .
$$

Sea $z=\frac{2 T}{\Delta t}$, entonces usando la notación dada en $(9)$ para $G_{k, l}$, se tiene

$$
z^{3}-G_{k, l}^{2} z=0
$$


Esta ecuación cúbica posee las siguientes raices:

$$
\begin{gathered}
z_{0}=0, \\
z_{+}=G_{k, l}, \\
z_{-}=-G_{k, l},
\end{gathered}
$$

observando el hecho que

$$
z=\frac{2 T}{\Delta t}=2 \frac{\tan \left(-w \frac{\Delta t}{2}\right)}{\Delta t}
$$

se tiene que $\lim _{\Delta t \rightarrow 0} z \longrightarrow-w$ cuando $U=V=0$. De este modo la matriz (24) converge a la matriz (8), con $U=V=0$. Esto muestraa que el esquema (20) es consistente. Tambien las matrices (24) y (8) tienen los mismos autovalores. Ademas los autovectores del sistema (20) son iguales a los autovalores $R_{+}, R_{-}, R_{0}$ del sistema analítico (7), cuando $U=V=0$.

Denotemos $S^{+}=\left(u^{+}, v^{+}, \phi^{+}\right)^{T}$. Entonces, se demuestra el siguiente resultado

Teorema 2.4 El esquema numérico (20) es consistente. Además, su solución general no estacionario es dado por

$$
\begin{gathered}
S^{+}(x, y, t)=\sum_{k=-\infty}^{\infty} \sum_{\substack{l=-\infty \\
(k, l) \neq(0,0)}}^{\infty}\left[\alpha_{+}(k, l) e^{i\left(w_{x} k x+w_{y} l y-z_{+} t\right)} R_{+}+\alpha_{-}(k, l) e^{i\left(w_{x} k x+w_{y} l y-z_{-} t\right)} R_{-}\right. \\
\left.+\alpha_{0}(k, l) e^{i\left(w_{x} k x+w_{y} l y-z_{0} t\right)} R_{0}\right]
\end{gathered}
$$

donde $z_{0}, z_{+}, z_{-}$son autovalores dados por (26), (27), (28) y $R_{0}(k, l), R_{+}(k, l), R_{-}(k, l)$ son los respectivos autovectores del sistema matricial (24) dados por (15), (17) y (18). Los coeficientes $\alpha_{+}(k, l), \alpha_{-}(k, l), \alpha_{0}(k, l)$ pueden ser determinados a partir de la condición inicial dada.

Por tanto los autovalores dados en (26), (27) y (28) convergen para los autovalores dados en (11), (12) y (13) respectivamente, cuando $\Delta t \rightarrow 0$ y $U=V=0$. Se observa que los autovalores de (26), (27) y (28) no dependen de $\Delta x$ y $\Delta y$, ellos apenas depende de $\Delta t$.

Las frecuencias (espacial y temporal) de onda son dados por los autovalores del sistema, ver [3]. De ese modo, se muestra que el método espectral aplicado al sistema de ecuaciones de aguas pòco profundas tiene una relación de disspersión en que la frecuencia de onda no depende de la posición espacial, o sea, de $\Delta x$ y $\Delta y$. Luego la velocidad de propagación también no depende de la posición espacial. Los modos $R_{0}(k, l), R_{+}(k, l), R_{-}(k, l)$ también no dependen de $\Delta x$ y $\Delta y$. Además en el método espectral, las redes diferentes no interfieren en la velocidad de propagación de la onda.

Se puede ver fácilmente que el sistema de ecuaciones de aguas poco profundas discretizado converge hacia su forma analítica, pues

$$
\begin{aligned}
& \frac{\beta^{+}-\beta^{-}}{\Delta t}=\frac{\beta(x, y, t+\Delta t)-\beta(x, y, t)}{\Delta t} \\
& \frac{\beta_{x}^{+}-\beta_{x}^{-}}{2}=\frac{\beta_{x}(x, y, t+\Delta t)-\beta_{x}(x, y, t)}{2} \\
& \frac{\beta_{y}^{+}-\beta_{y}^{-}}{2}=\frac{\beta_{y}(x, y, t+\Delta t)-\beta_{y}(x, y, t)}{2}
\end{aligned}
$$


donde $\beta=u, v$ y $\phi$. De este modo, cuando $\Delta t \rightarrow 0$ las expresiones (28), (29) y (30), convergen para

$$
\begin{aligned}
& \frac{\beta^{+}-\beta^{-}}{\Delta t} \rightarrow \frac{\partial \beta}{\partial t}(x, y, t) \\
& \frac{\beta_{x}^{+}-\beta_{x}^{-}}{2} \rightarrow \frac{\partial \beta}{\partial x}(x, y, t) \\
& \frac{\beta_{y}^{+}-\beta_{y}^{-}}{2} \rightarrow \frac{\partial \beta}{\partial y}(x, y, t)
\end{aligned}
$$

donde $\beta=u, v$ y $\phi$.

Luego, utilizando (31), (32) y (33) en el sistema de ecuaciones de aguas poco profundas discretizada, se tiene que éste es convergente.

\section{Conclusión}

Este estudio muestra una ventaja del método espectral en relacion al metodo de diferencias finitas en el estudio de las ecuaciones de aguas poco profundas, pues no hay interferencia de la red cuando se usa el método numérico espectral en el análisis de propagación de las ondas de aguas poco profundas, diferentemente del método de diferencias finitas, entonces al ser usado el método espectral cualquier interferencia en la propagación de la onda se debe a algún otro factor.

\section{Referencias bibliográficas}

[1] Briggs, L., Henson E. (1995). The DFT: An Owners' Manual for the Discrete Fourier Transform. Philadelphia: Society for industrial and Applied Mathematics.

[2] Chorin, J., Marsden, E. (1992). A Mathematical Introduction to Fluid Mechanics. New York: Springer-Verlag.

[3] Longuet-Higgins, M. S. (1968). The Eigenfunctions of Laplace's Tidal equations over a Sphere. The Royal Society, 262 (1132), 511-607.

[4] Elvius, T., Sundstrom, A. (1973). Computationally effective schemes and boundary conditions for a fine barotropic model based on shallow-water equations. Tellus XXV, 132-155.

[5] Ritchie, H. (1998). Application of the Semi-Lagrangean Method to a Spectral Model of the Shallow Water Equations. Monthly Weather Review, 116, 1587-1598. 